\title{
Difference in basic concept of coronary bifurcation intervention between Korea and Japan. Insight from questionnaire in experts of Korean and Japanese bifurcation clubs
}

\author{
Yoshinobu Murasato $^{1}$ (D) $\cdot$ Yoshihisa Kinoshita ${ }^{2} \cdot J_{\text {Jnya Shite }}^{3} \cdot$ Yutaka Hikichi $^{4} \cdot$ Chang-Wook Nam $^{5} \cdot$ Bon-Kwon Koo $^{6}$
}

Received: 13 September 2020 / Accepted: 30 November 2020 / Published online: 16 January 2021

(c) The Author(s) 2021

\begin{abstract}
The coronary bifurcation intervention varies among countries due to the differences in assessment of lesion severity and treatment devices. We sought to clarify the difference in basic strategy between South Korea and Japan. A total of 19 and 32 experts from Korean (KBC) and Japanese Bifurcation Clubs (JBC), respectively, answered a survey questionnaire concerning their usual procedure of coronary bifurcation intervention. JBC experts performed less two-stent deployment in the left main (LM) bifurcation compared to KBC experts (JBC vs. KBC: median, $1-10 \%$ vs. $21-30 \%, p<0.0001$ ) instead of higher performance of side branch dilation after cross-over stenting in both LM (60\% vs. 21\%, $p=0.001)$ and non-LM bifurcations ( $30 \%$ vs. $5 \%, p=0.037$ ). KBC experts more frequently performed proximal optimization technique (POT) in non-LM bifurcation $(41-60 \%$ vs. $81-99 \%, p=0.028)$ and re-POT in both LM (1-20\% vs. $81-99 \%, p=0.017)$ and non-LM bifurcations $(1-20 \%$ vs. $81-99 \%, p=0.0003)$. JBC experts more frequently performed imaging-guided percutaneous coronary intervention, whereas KBC experts more often used a pressure wire to assess side branch ischemia. JBC experts used a rotablator more aggressively under the guidance of optical coherence tomography. We clarified the difference in the basic strategy of coronary bifurcation intervention between South Korea and Japan for better understanding the trend in each country.
\end{abstract}

Keywords Bifurcation · Drug-eluting stent · Fractional flow reserve · Intravascular ultrasound · Optical coherence tomography

Yoshinobu Murasato

y.murasato@gmail.com

1 Department of Cardiology and Clinical Research Center, National Hospital Organization Kyushu Medical Center, 1-8-1, Jigyohama, Chuo, Fukuoka 810-8563, Japan

2 Department of Cardiovascular Medicine, Toyohashi Heart Center, Toyohashi, Japan

3 Department of Cardiology, Osaka Saiseikai Nakatsu Hospital, Osaka, Japan

4 Department of Cardiology, Saga University, Saga, Japan

5 Division of Cardiology, Keimyung University Dongsan Hospital, Daegu, South Korea

6 Department of Internal Medicine and Cardiovascular Center, Seoul National University Hospital, Seoul, South Korea

\section{Introduction}

Providing provisional treatment and reducing two-stent deployment for coronary bifurcation lesions has gained world-wide acceptance to avoid unnecessary treatment and reduce the risk of adverse cardiac events [1-3]. In addition, proximal optimization technique (POT) has been widely used instead of routine kissing balloon inflation (KBI) for the optimal dilation of proximal main vessels (MV) $[1,2]$. However, interventions for coronary bifurcation remain challenging because large variations in lesion anatomy, method of assessment of lesion severity, and treatment devices make standardization of treatment difficult. The frequency of twostent deployment, POT and KBI, penetration under imagingor physiologic-guidance, and usage of atherectomy devices still widely vary depending on the operator's skill and judgment, institution-specific practices, and social circumstance.

In Asia, South Korea and Japan lead in the number of coronary interventions performed annually, and both imagingand physiologic-guidance are popular in these countries. 
However, these countries follow different practices for bifurcation treatment probably due to differences in concept or philosophy.

We sought to investigate the basic strategy of coronary bifurcation treatment using a questionnaire answered by experts in the Japanese Bifurcation Club (JBC) and Korean Bifurcation Club (KBC) and to clarify the differences between the practices followed in these countries.

\section{Methods}

We prepared both paper and internet survey questionnaires for usual bifurcation treatment in left main (LM) and nonLM lesions with side branch (SB) diameter $\geq 2.5 \mathrm{~mm}$. The questionnaire included the following items: background of percutaneous coronary intervention (PCI) operator and institute, two-stent deployment, POT/re-POT, method of SB dilation, status of usage of the devices, imaging-guidance, and rotablation (Table 1). Thirty-two and 19 experts in JBC and $\mathrm{KBC}$ answered the questionnaire, respectively.

\section{Statistical analysis}

Data were presented as median for discrete variables and ordinal scales. Discrete variables were compared between the groups using a Fisher's exact test, while ordinal scales were compared using a Mann-Whitney $U$ test. All $p$ values were two-sided and considered statistically significant at levels $<0.05$. All statistical analyses were performed with EZR software (Saitama Medical Center, Jichi Medical University, Saitama, Japan), a modified version of $\mathrm{R}$ commander (The R Foundation for Statistical Computing, Vienna, Austria).

\section{Results}

\section{Background of $\mathrm{PCl}$ operator and institute}

JBC experts belonged to an institute wherein few PCI cases were handled compared to the institutes where the $\mathrm{KBC}$ experts were employed (median: JBC 301-700 cases/institute vs. KBC 1001-2000 cases/institute, $p<0.0001$ ). The annual PCI cases handled by an expert were less in Japan, compared to South Korea (JBC 101-200 cases vs. KBC 201-300 cases, $p=0.022$ ). Conversely, the experts from Japan had a longer tenure than the experts from South Korea $(p=0.002, \geq 21$ years; JBC $32 \%$ vs. KBC 0\%) (Fig. 1).

\section{Two-stent deployment}

Two-stent deployment was performed more often for LM bifurcation among KBC experts (JBC $1-10 \%$ vs. KBC
$21-30 \%, p<0.0001)$, whereas the number was not significantly different between the groups for non-LM bifurcation (Fig. 2a). The most and second most frequently used two-stent techniques were completely different, which were culotte $(81 \%)$ and T-stenting (45\%) in JBC experts and crush stenting in both groups (53\% and 37\%) in $\mathrm{KBC}$ experts $(p<0.0001)$ (Fig. 2b).

\section{POT/re-POT}

Although routine performance of the POT in the LM bifurcation was numerically more frequent in $\mathrm{KBC}$ experts (JBC $26 \%$ vs. KBC $47 \%$ ), there was no statistically significant difference. The POT performance in the non-LM bifurcation was more in KBC experts (JBC 41-60\% vs. KBC 81-99\%, $p=0.028$ ) (Fig. 3a). In terms of the re-POT, KBC experts performed more frequently in both LM (JBC 1-20\% vs. KBC 81-99\%, $p=0.017$ ) and non-LM bifurcations (JBC $1-20 \%$ vs. KBC $81-99 \%, p=0.0003$ ) (Fig. 3b).

\section{SB dilation}

The incidence of SB dilation after cross-over stenting was higher in JBC experts in both LM (JBC 81-99\%, KBC $21-40 \%, p<0.0001$ ) and non-LM bifurcations (JBC $61-80 \%$, KBC $1-20 \%, p=0.004$ ) (Fig. 3c). Kissing balloon inflation (KBI) was most frequently used in both LM and non-LM bifurcations in both groups of experts (Fig. 3d). In the case of a negative value of fractional flow reserve (FFR)/ instantaneous wave-free ratio (iFR) in the stent-jailed SB, more JBC experts thought SB dilation with KBI or simple dilation was necessary in LM (JBC $60 \%$ vs. KBC $21 \%$, $p=0.001$ ) and non-LM bifurcations (JBC 30\% vs. KBC 5\%, $p=0.037$ ), while $\mathrm{KBC}$ experts made the negative results of physiological assessment more important to reduce SB dilatation (Fig. 4).

\section{Device (balloon, guide wire, imaging device, pressure wire, and microcatheter/double lumen catheter)}

In the LM bifurcation, JBC experts used more guide wires (JBC 3 vs. $\mathrm{KBC} 2, p<0.0001$ ) and double lumen catheters routinely (JBC $75 \%$ vs. KBC $11 \%, p<0.0001$ ), whereas routine use of pressure wires for the assessment of the jailed SB stenosis was less frequent (JBC 3\% vs. KBC 32\%, $p=0.008)$. In the non-LM bifurcation, a similar trend was found: JBC experts used more guide wires (JBC 3 vs. KBC $2, \mathrm{p}=0.002)$ and double lumen catheters (JBC $71 \%$ vs. KBC $5 \%, p<0.0001$ ), with less routine use of pressure wires (JBC $3 \%$ vs. KBC $21 \%, p=0.06$ ) (Fig. 4). 


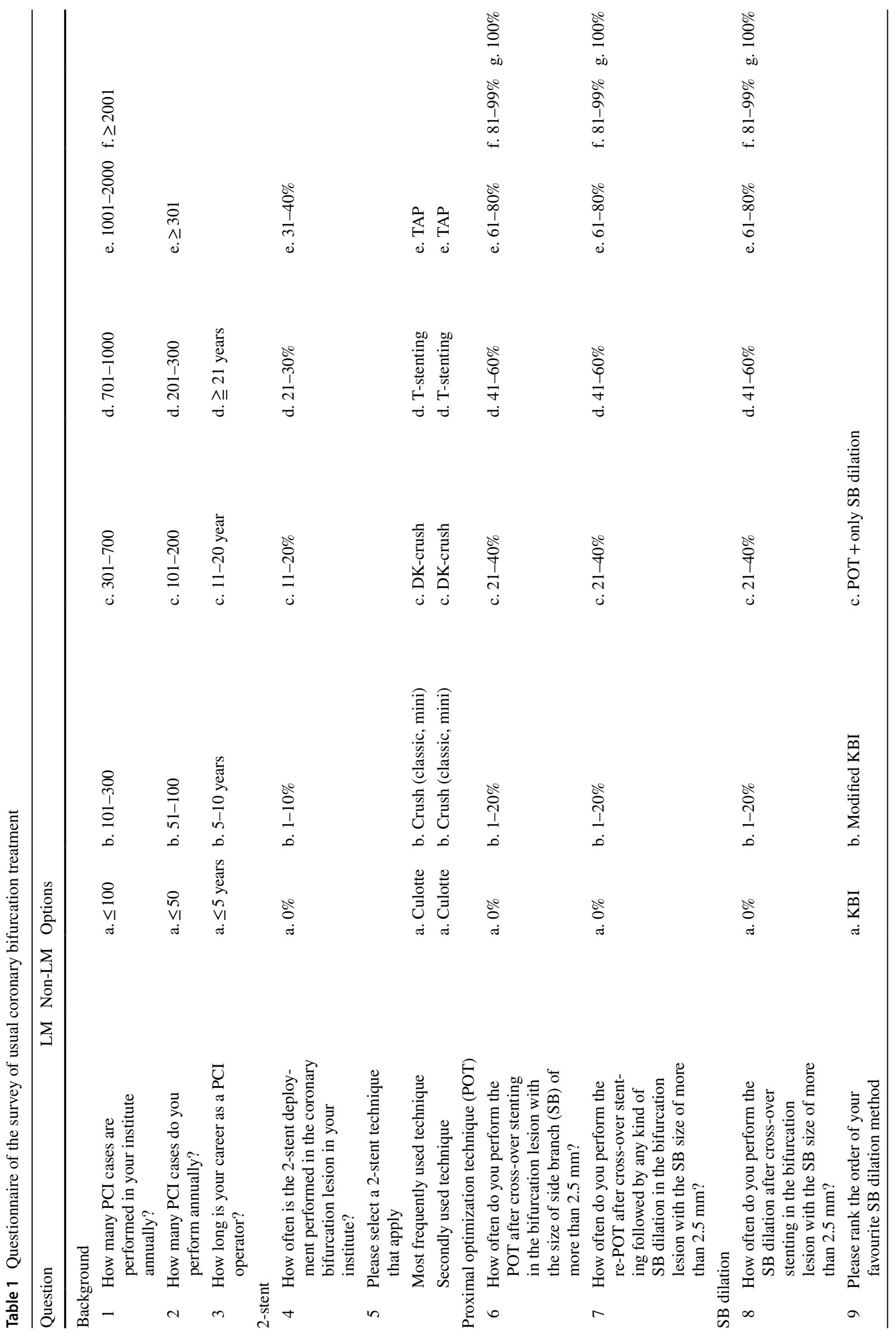




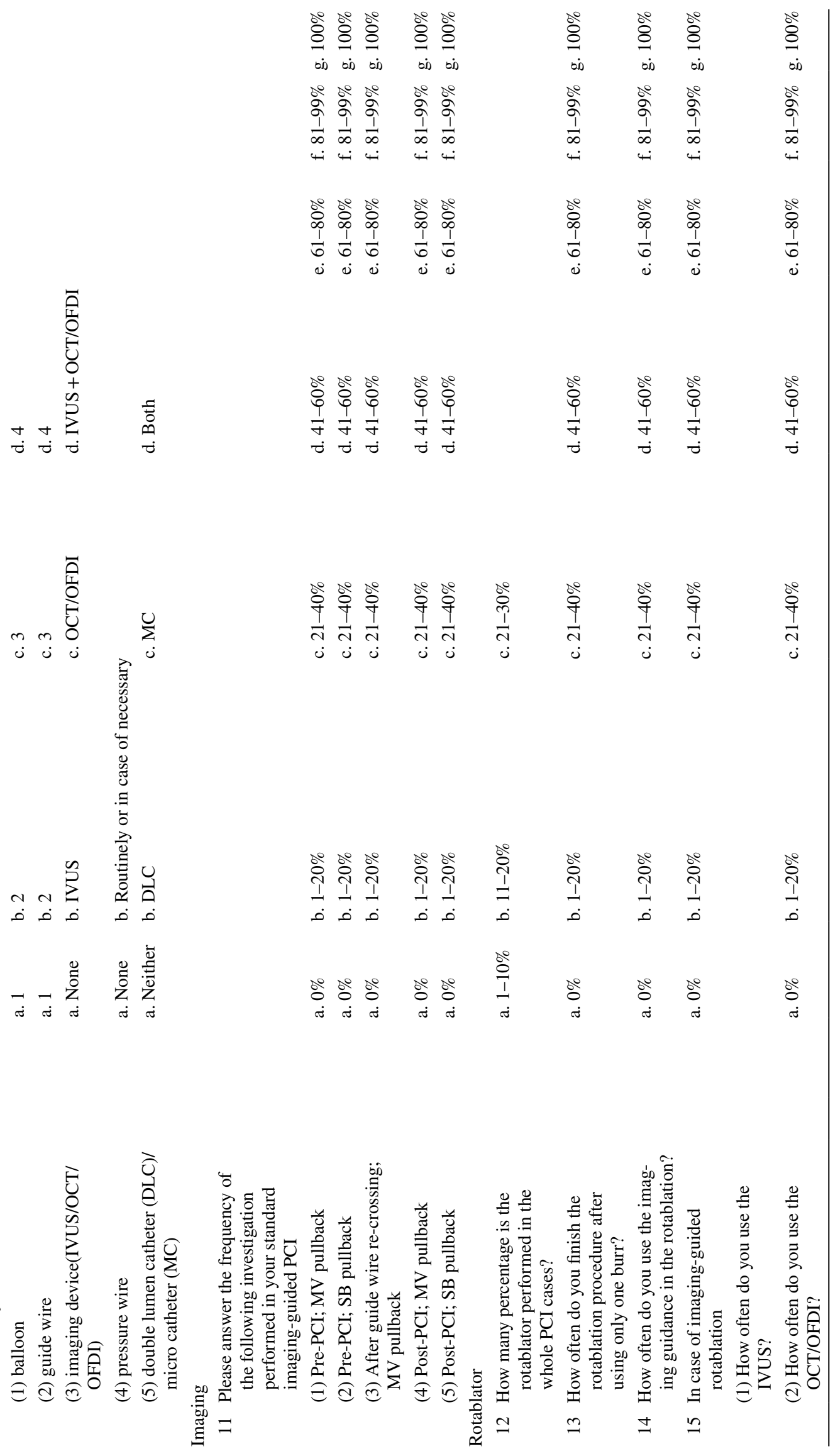


Imaging guidance (device and timing)

As for imaging device, JBC experts usually used intravascular ultrasound (IVUS) or optical coherence tomography (OCT)/optical frequency domain imaging (OFDI), which was more frequently in the LM bifurcation (JBC 94\% vs. $\mathrm{KBC} 63 \%, p=0.0004)$. JBC experts used OCT/OFDI in about $40 \%$ in both LM and non-LM bifurcations, while KBC experts never primarily used them. In the LM bifurcation, pullbacks from main vessels (MV) in both pre- $(p=0.0005)$ and post-PCI $(p=0.019)$ were popular in both groups of experts; however, JBC experts performed this technique more frequently. Pullbacks from SB in both pre- and postPCI were performed less than those in MV, and frequencies were not significantly different between JBC and KBC experts. However, assessment of guide wire re-crossing point to the SB was more frequently performed in JBC experts (JBC $100 \%$ vs. KBC $1-20 \%, p<0.0001$ ). In the nonLM bifurcation, $\mathrm{KBC}$ experts perform less assessment of the MV in both pre- $(p=0.0005)$ and post-PCI $(p=0.003)$ as well as that of the guide wire re-crossing point (JBC 100\% vs. $\mathrm{KBC} 1-20 \%, p=0.0002$ ) (Fig. 5).

\section{Rotablation (frequency, burr size, imaging-guidance, and SB treatment)}

Rotablation was more frequently used in $\mathrm{JBC}$ experts (JBC $11-20 \%$ vs. KBC $1-10 \% \%, p=0.01$ ). JBC experts performed rotablation under the imaging guidance more frequently (JBC $100 \%$ vs. KBC $41-60 \%, p<0.0001)$ with more OCT/OFDI guidance $(p=0.0003)$, while KBC experts mainly used the IVUS $(p=0.003)$. JBC experts used rotablation more aggressively for SB plaque modification (JBC $1-5 \%$ vs. $\mathrm{KBC} 0 \% \%, p=0.0003$ ) (Fig. 6).

\section{Discussion}

The present study clarified the difference in basic concept of coronary bifurcation treatment between JBC and $\mathrm{KBC}$ experts.

\section{Social background}

There were more small-volume PCI centres and few operator's personal annual cases among JBC experts; however, they had a longer operator's career. Intensification to the large cardiovascular centre has been advanced in South Korea, which leads to more experience in younger operators. Physiological assessment using a pressure wire and imaging device during PCI are reimbursed by social insurance in Japan, whereas this is not done in South Korea. Penetration of imaging-guidance is more than $90 \%$ in daily practice in 
Japan

\section{A Institutional PCl cases}

\section{B Personal PCl cases}

\section{Personal career}

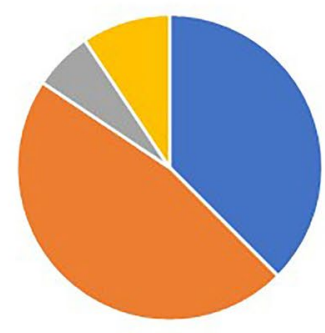

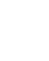

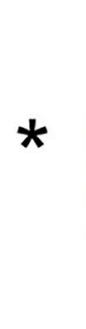

Korea
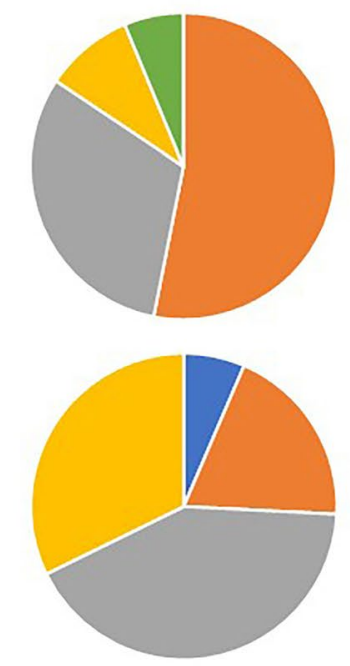
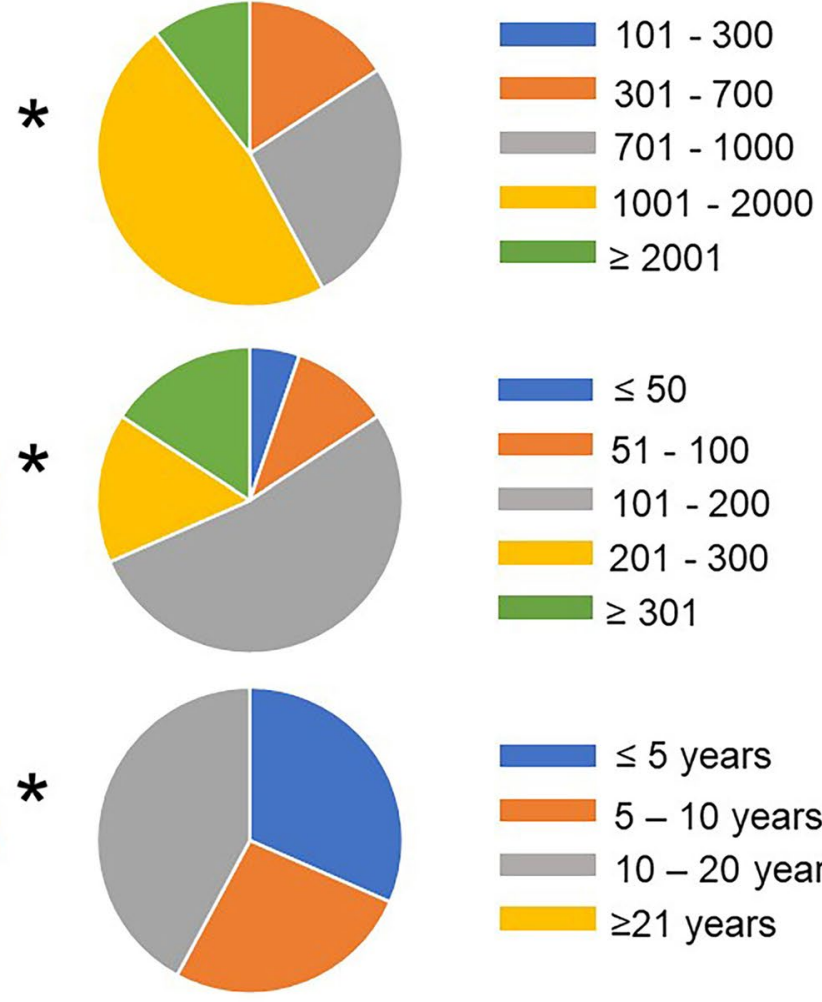

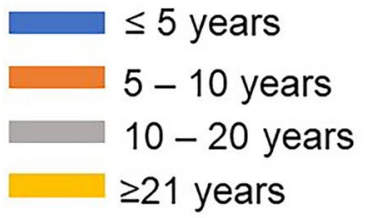

Fig. 1 Comparison between experts of Japanese Bifurcation Club (JBC) and Korean Bifurcation Club (KBC) in terms of background. a Institutional annual PCI cases. b Personal annual PCI cases. c Personal career as a PCI operator. * $p<0.05$ in comparison of JBC vs. KBC

Japan [the annual report in 2014 of the Japanese Registry of All Cardiac and Vascular Disease (http://www.j-circ.or.jp/ jittai_chosa/jittai_chosa2014web.pdf)], while 39\% in Korean coronary bifurcation registry (COBIS) II [4]. The difference in imaging-guidance in daily practice may have some influence on basic concept.

\section{Two-stent deployment}

Two-stent deployment is more frequently used by KBC experts, which is within the acceptable range in comparison with pivotal international studies [5-8], while JBC experts strictly limit two-stent deployment. Since frequency of true bifurcation lesion does not seem to differ between Japan and South Korea, the difference in frequency of two-stent deployment may mainly attribute to their response to prePCI SB lesion severity (stenosis and lesion length) and that to the condition after SB dilation (remained stenosis and dissection). Although KBC experts perform more physiological assessment in the SB and leave the SB without any balloon dilation more than JBC experts, more frequency of two-stenting in South Korea indicates more performance of provisional or planned two-stenting once they decide to treat the SB. Angiographically hazy image in the SB without imaging guidance is likely to induce more performance of two-stenting in Korea where $60 \%$ of bifurcation PCI is not performed under the imaging guidance, while mild dissection clearly detected by the imaging is likely to be left without stenting in Japan with higher penetration of the imaging guidance. In the DEFINITION trial, two-stenting brought better survival-free rate from adverse cardiac events compared to provisional stenting in the complex true bifurcation lesion [8]. Conversely, many randomized trials with comparison between provisional stenting and two-stenting demonstrated worse $[9,10]$ or ineffective outcomes after two-stenting [11, 12] except for DK-crush V study [13]. The proportion of 2-stent deployment has been decreased in both Japan (29.6\% [14] to 21\% [15]) and South Korea (40.3\% [4] to $23.2 \%$ [16]), because more adverse cardiac events were observed in their initial experience of 1st-generation DES $[14,17]$. Favorable clinical outcome of 2 -stent with newgeneration DES similarly as that of provisional stenting has been reported in Korean registry [17] but not in Japanese registry [15]. Japanese experts have continued more effort so as to leave more mildly dissected SB without stenting, to treat with drug-coated balloon and to ablate the SB plaque 


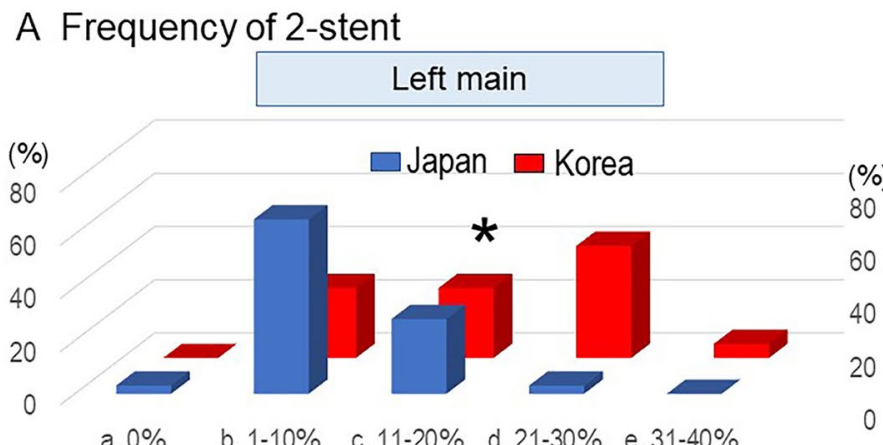

$\begin{array}{lllll}\text { a. } 0 \% & \text { b. } 1-10 \% & \text { c. } 11-20 \% & \text { d. } 21-30 \% & \text { e. } 31-40 \%\end{array}$

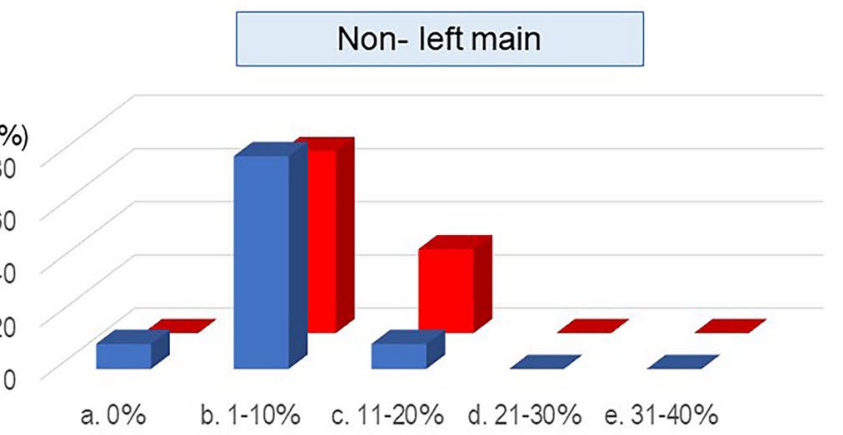

(2) Secondly use
(1) Most frequently use

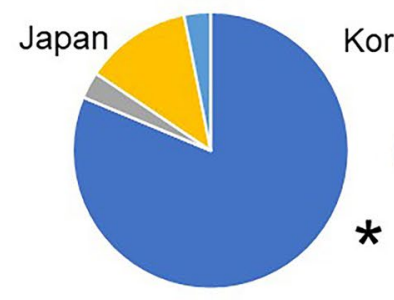

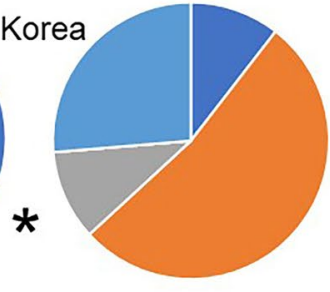

Culotte

Crush (classic, mini)

DK-Crush

T-stenting

TAP

Others

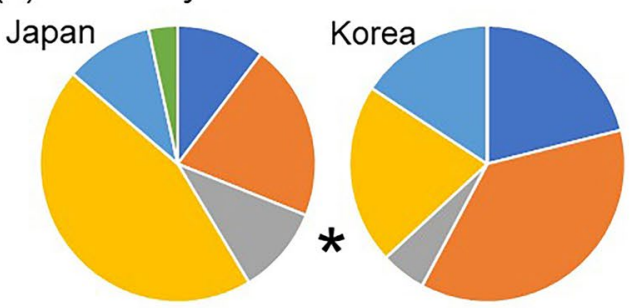

Fig. 2 Comparison between JBC and KBC experts in 2-stent technique. a Frequency of 2-stent in left main (left) and non-left main bifurcations (right). b 2-stent technique. (1) Most frequently used technique. (2) Secondly used technique. ${ }^{*} p<0.05$ in comparison of JBC vs. KBC

A POT

C SB dilation after cross-over stenting
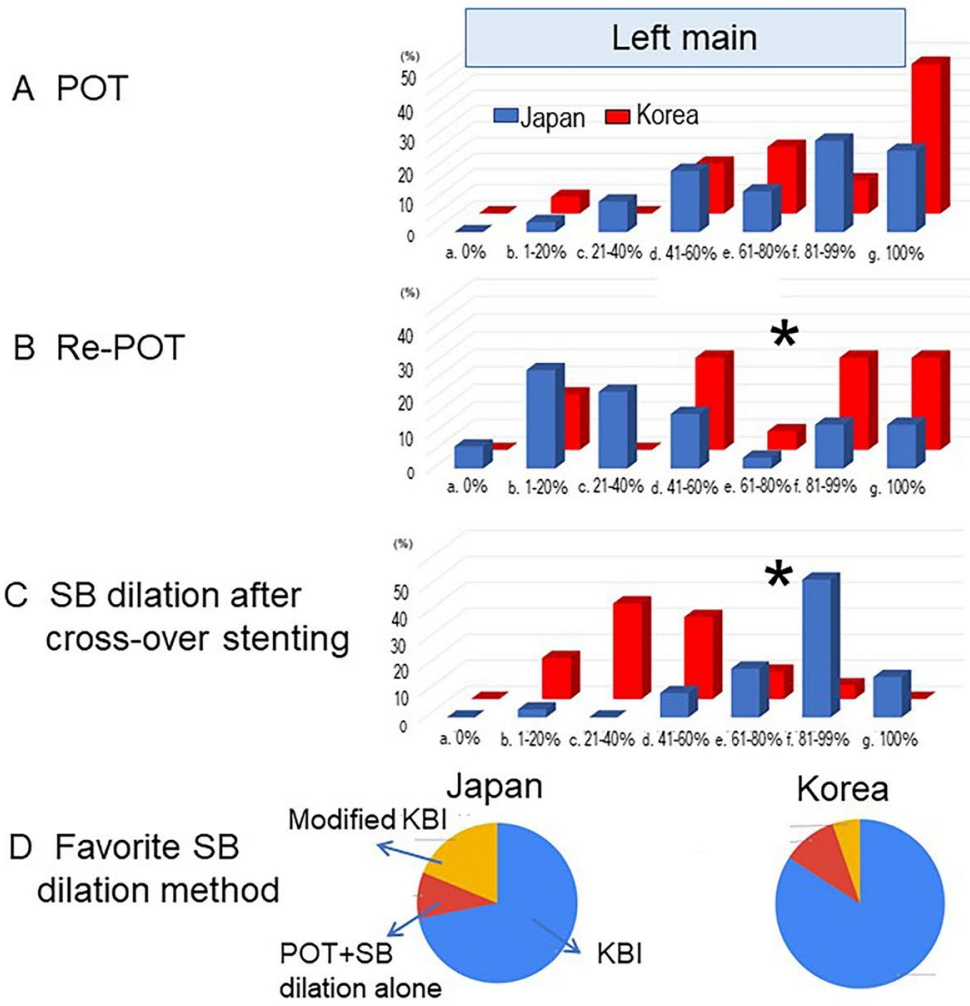

Fig. 3 Comparison between JBC and KBC experts in proximal optimization technique (POT)/re-POT and side branch (SB) dilation in cross-over stenting. a Frequency of POT in left main (left) and non- left main bifurcations (right). b Frequency of re-POT. c Frequency of SB dilation. d Favourite method of SB dilation. KBI kissing balloon inflation. $* p<0.05$ in comparison of JBC vs. KBC 


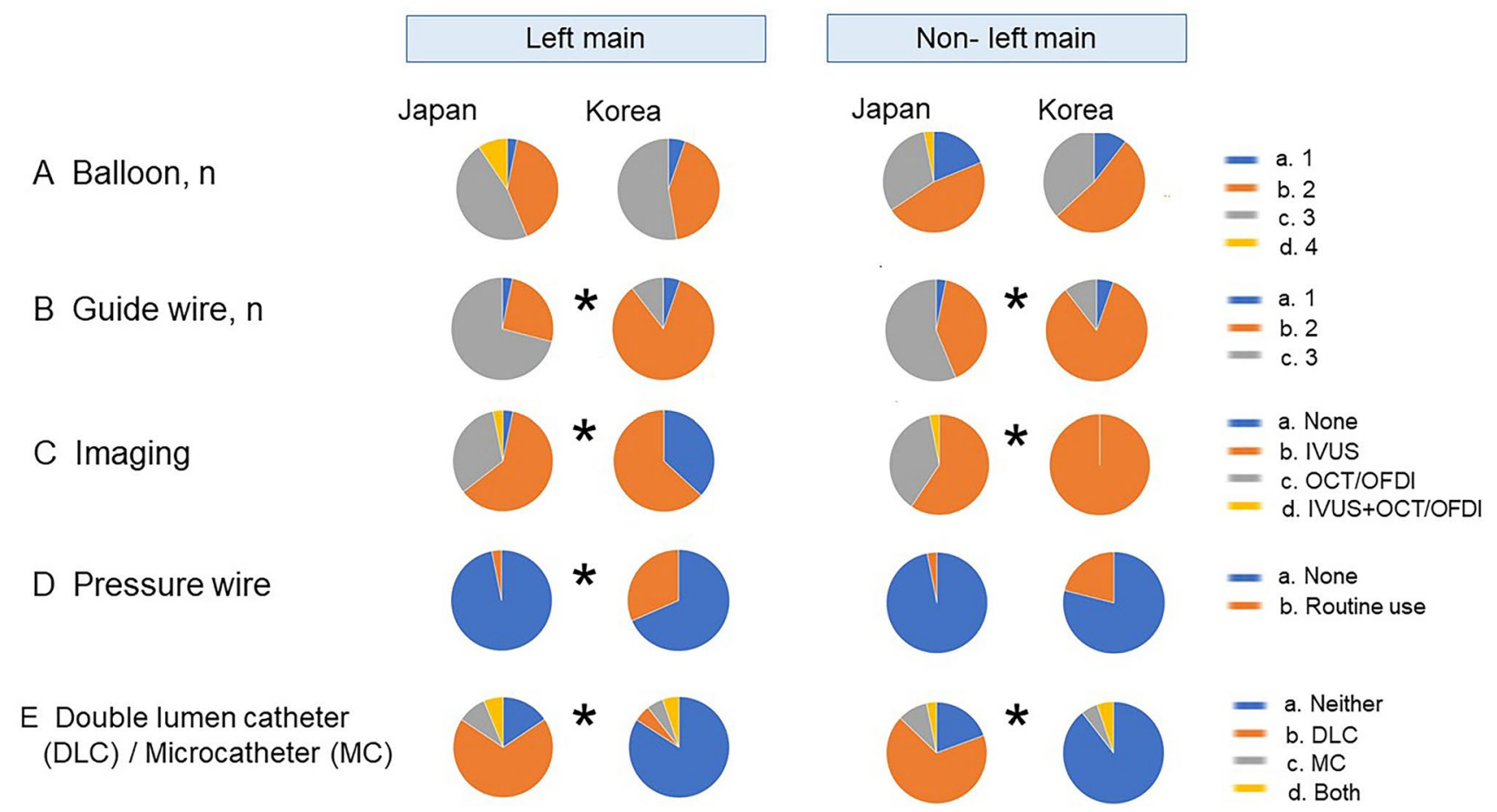

Fig. 4 Comparison between JBC and $\mathrm{KBC}$ experts in routine use of the devices for usual cross-over stenting. a Number of balloons. $\mathbf{b}$ Number of guide wires. c Imaging device. IVUS intravascular ultrasound, $O C T$ optical coherence tomography, OFDI optical frequency

domain imaging. d Use of pressure wire. e Use of double lumen catheter (DLC) and/or microcatheter (MC). ${ }^{*} p<0.05$ in comparison of JBC vs. KBC

for the reduction of SB stenting. However, these attempts have not yet been investigated in a large-scale study and ideal proportion of 2-stent providing both clinical and cost benefits is still argued.

The primary stenting technique was culotte stenting in JBC experts and crush stenting in KBC experts. In Korean registries, crush stenting was most frequently used in $41-53 \%$ and culotte stenting was less used in $2-12 \%$ [4, 18]. On the contrary, there was higher prevalence of culotte stenting in Japan (16-81\%) [14, 19]. After introducing newgeneration DES, TLR was dramatically reduced in Korea (16.1-7.8\%) [17] and Japan (13.6-6.7\%) [14, 19]. Culotte stenting provides a certain DES coverage in the whole bifurcation with less layer; however, there are risks of MV or SB occlusion during the procedure as well as inadequate expansion of branch ostium due to jailed strut [20-22]. Crush stenting has a much lower risk of branch loss due to its protective procedure for MV occlusion and a certain SB stenting; however, there remain three-layered overlapping struts in the proximal MV and difficulty in SB opening [20-22]. In the DK-crush III study, randomized comparison was done between DK-crush and culotte stentings in unprotected LM bifurcation, and DK-crush stenting was superior in free survival from major adverse cardiac events and target lesion revascularization [23]. However, a meta-analysis comparing

between culotte and crush stentings did not show superiority in either of them [24]. Since both 2-stenting required specific complicated procedures, performing with operator's familiar technique may lead to more optimal procedure and better clinical outcome.

\section{POT/re-POT}

POT can provide symmetric adequate stent expansion in the proximal MV with appropriate widening of the jailed struts at the SB ostium, which facilitates optimal guide wire re-crossing to the distal cell for the SB dilation [25, 26]. Both JBC and KBC experts realized the efficacy of the $\mathrm{POT}$ and performed it with higher frequency. Re-POT is recommended to correct the stent deformation or malapposition induced by KBI or SB dilation [25]; however, a recent study demonstrated risks of worsening SB obstruction [27] and stent deformation by insertion of the re-wrapped POT balloon. KBC experts performed more re-POT, while JBC experts does not aggressively. JBC experts generally considered that re-POT is not mandatory, when adequate stent expansion without any significant malapposition or stent deformation is confirmed in the imaging [28, 29]. Re-POT is an essential procedure in angio-guided bifurcation PCI to secure adequate stent expansion without malapposition. 
A Pre-PCl MV pullback

SB pullback

B After GW MV pullback
recrossing

C Post-PCl MV pullback

SB pullback
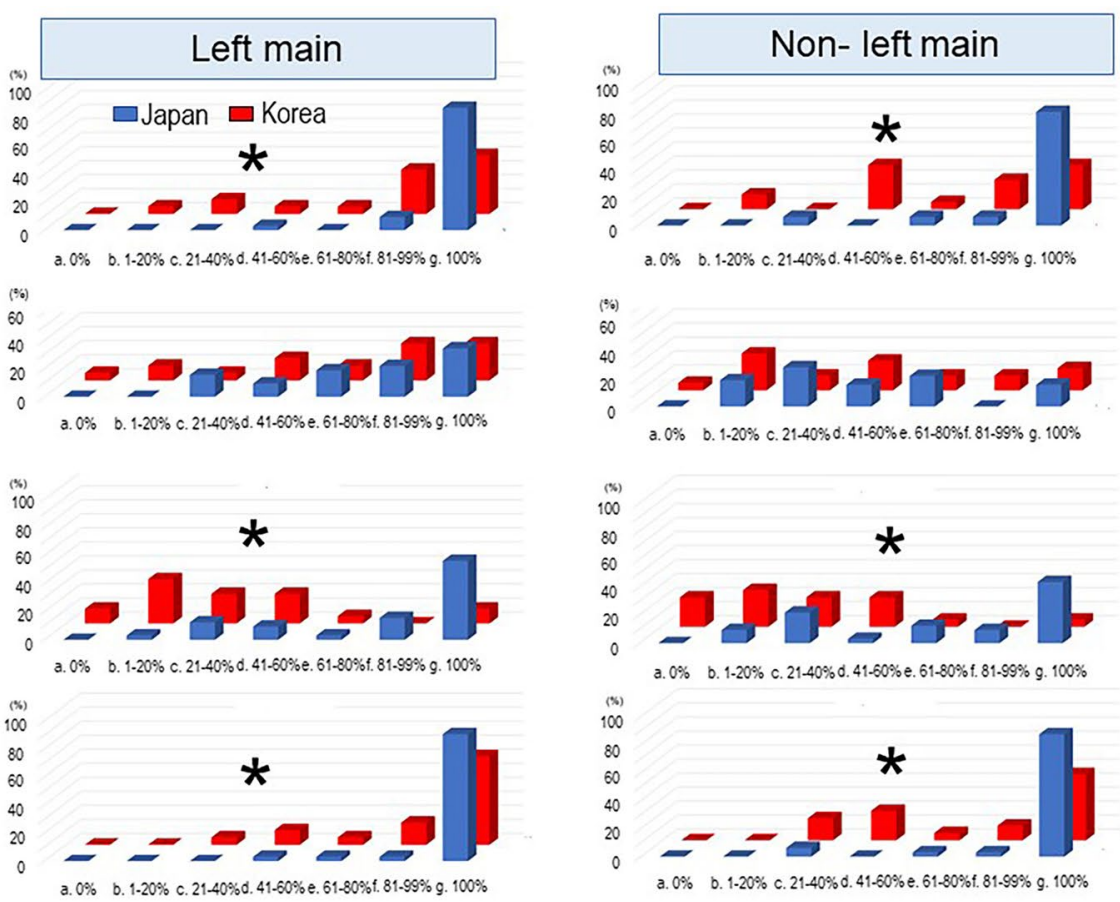

$(5)$
100
80
60
40
20
0
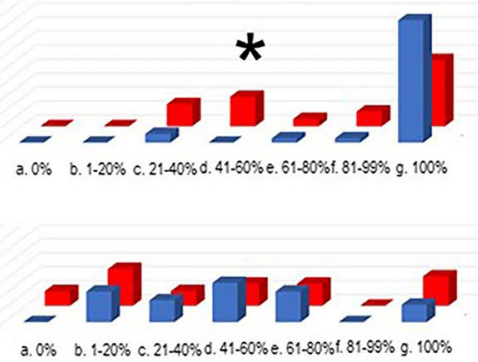

Fig. 5 Comparison between JBC and KBC experts in frequency of the imaging observation in left main (left) and non-left main bifurcations (right). a Pre-PCI. Main vessel (MV, upper) and side branch

\section{SB dilation according to physiological assessment versus imaging guidance}

JBC experts performed SB dilation after cross-over stenting more aggressively in both LM and non-LM bifurcation lesions with large SB $(\geq 2.5 \mathrm{~mm})$ as shown in Fig. 3c, while KBC experts assessed more FFR/iFR and treated the SB more strictly according to its results as shown in Fig. 4d. FFR measurement in the jailed SB revealed that $70 \%$ of angiographically significant stenosis did not present a significant FFR value $(<0.80)$ [30], and FFR-guided deferring SB treatment did not show worsening of adverse cardiac events at long-term follow-up [31, 32]. Korean experts are more unlikely to perform SB dilation according to their experience of physiology-guided deferring of the jailed SB treatment [30-32]. However, in the bench testing and computer simulation, jailed struts at the SB ostium introduces flow turbulence and generates a low shear stress area [33], which is likely to provoke atherosclerotic change. The OCT observation of the jailed SB without KBI treatment at 9-12-month follow-up revealed
(SB) pullbacks (lower). b After guide wire (GW) re-crossing. c Post-PCI. Main vessel (MV, upper) and side branch (SB) pullbacks (lower). ${ }^{*} p<0.05$ in comparison of JBC vs. KBC

more thrombus attachment compared to the opened SB with KBI [34]. A greater number of jailed struts in the SB ostium was associated with SB luminal narrowing at follow-up [35]. JBC experts used more OCT/OFDI and confirmed the guide wire re-crossing point in the imaging more frequently with more usage of the double lumen catheter. Hence, more Japanese experts focus on optimal SB dilation with clearance of jailed struts under the practical guidance of the imaging, which results in more SB dilation and less SB stenting. Since most of the previous reports were based on angio-guided procedure, suboptimal KBI which occupied more than $50 \%$ of the cases in the 3-dimentional OCT observation [36] has a potential risk of adverse cardiac events. Physiology-guided SB treatment is a smart strategy to reduce the complicated procedure and the stent failure induced by SB dilation which are hardly detected in the angiography. Imaging-guidance with high resolution, 3-dimentional OCT/OFDI mainly investigated in Japan, has a great potential to correct stent failure and lead to optimal procedure. Further study is warranted for its impact on long-term clinical outcome. 


\section{A Frequency of rotablation to whole $\mathrm{PCl}$}

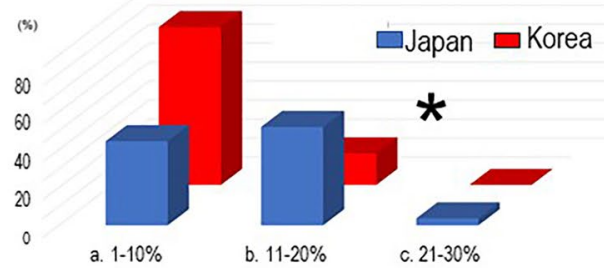

B Rotablation with one burr alone

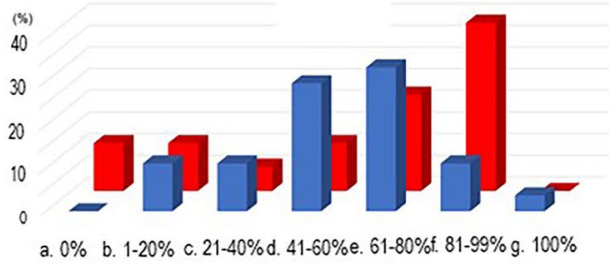

C Rotablation for SB lesion modification

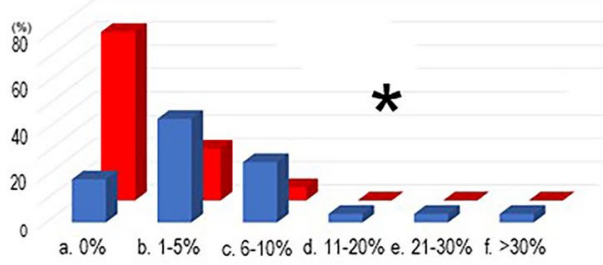

Fig. 6 Comparison between JBC and $\mathrm{KBC}$ experts in rotablation. a Frequency of rotablation to whole PCI. b Frequency of rotablation with one burr alone. c Frequency of rotablation for SB lesion modi-

\section{Role of rotablation in bifurcation $\mathrm{PCI}$}

JBC experts performed more rotablation with a greater number of the burr under complete imaging-guidance. Since the calcification in the bifurcation has risks of stent underexpansion, carina shift, and SB occlusion [37], adequate ablation with rotablator is required. The OCT/OFDI can clearly illustrate the border of calcification and indicate residual thickness after rotablation accurately. Calcium thickness $<500-700 \mu \mathrm{m}$ allows a scoring balloon to make crackles for adequate stent expansion [38,39], which is the target index during the rotablation. Since the IVUS which Korean experts mainly use is inferior to the OCT/OFDI in the illustration of calcification, aggressive rotablation is unlikely to be promoted.

\section{Study limitations}

The present study had some limitations. (1) Since this survey was performed on the selected experts of $\mathrm{KBC}$ and $\mathrm{JBC}$, their therapeutic concept might be more specialized compared to nationwide average strategy. (2) Although there

\section{Rotablation under imaging-guidance}
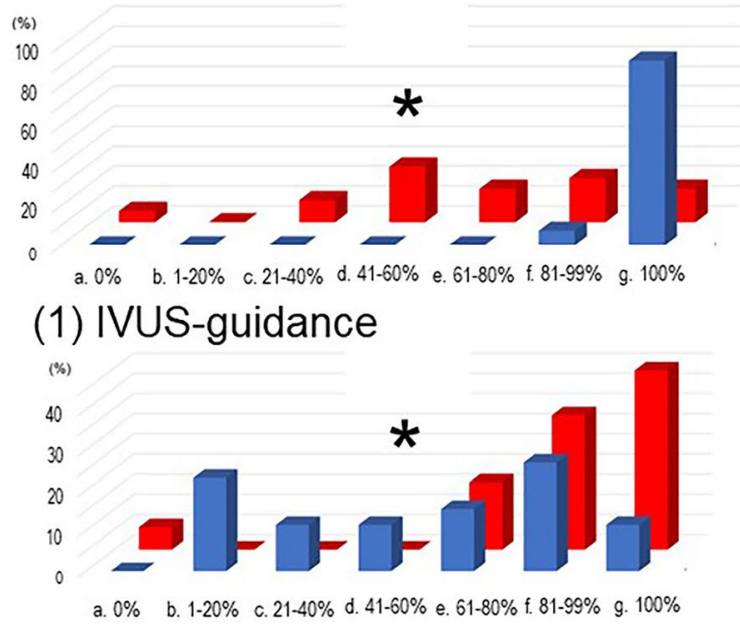

(2) OCT/OFDI-guidance

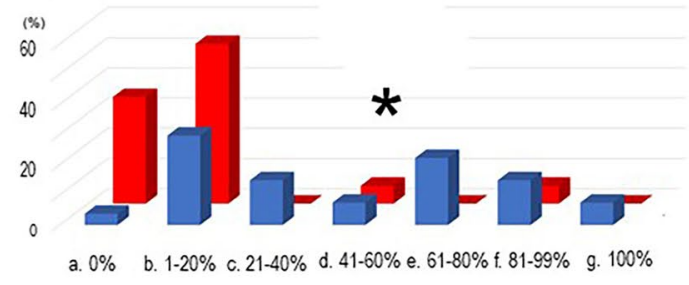

fication. d Rotablation under imaging-guidance. (1) Frequency of IVUS-guidance. (2) Frequency of OCT/OFDI-guidance. ${ }^{*} p<0.05$ in comparison of JBC vs. KBC

are significant statistical differences between $\mathrm{KBC}$ and JBC experts in several points, the small sample size might include some bias.

\section{Conclusion}

JBC experts perform imaging-guidance more strictly at preand post-PCI as well as after MV stenting for the optimization of the bifurcation PCI. Moreover, they use rotablator more aggressively under OCT/OFDI guidance, whereas KBC experts mostly use FFR/iFR measurement and POT/ re-POT which are currently recommended. JBC experts perform less 2-stenting instead of more performance of SB dilation after cross-over stenting. The most popular 2-stenting is different, that is, culotte stenting in JBC and crush stenting in $\mathrm{KBC}$ experts. Interventions for coronary bifurcation vary among the countries due to differences in social background and whether the basic strategy is determined based on the findings of angiography, imaging, or physiology.

Funding The study is not supported by any funding. 


\section{Compliance with ethical standards}

Conflict of interest Authors have no conflict of interest.

Open Access This article is licensed under a Creative Commons Attribution 4.0 International License, which permits use, sharing, adaptation, distribution and reproduction in any medium or format, as long as you give appropriate credit to the original author(s) and the source, provide a link to the Creative Commons licence, and indicate if changes were made. The images or other third party material in this article are included in the article's Creative Commons licence, unless indicated otherwise in a credit line to the material. If material is not included in the article's Creative Commons licence and your intended use is not permitted by statutory regulation or exceeds the permitted use, you will need to obtain permission directly from the copyright holder. To view a copy of this licence, visit http://creativecommons.org/licenses/by/4.0/.

\section{References}

1. Lassen JF, Holm NR, Banning A, Burzotta F, Lefèvre T, Chieffo A, et al. Percutaneous coronary intervention for coronary bifurcation disease: 11 th consensus document from the European Bifurcation Club. EuroIntervention. 2016;12:38-46.

2. Lassen JF, Burzotta F, Banning AP, Lefèvre T, Darremont O, Hildick-Smith D, et al. Percutaneous coronary intervention for the left main stem and other bifurcation lesions: 12th consensus document from the European bifurcation club. EuroIntervention. 2018;13:1540-53.

3. Burzotta F, Lassen JF, Louvard Y, Lefèvre T, Banning AP, Daremont $\mathrm{O}$, et al. European bifurcation club white paper on stenting techniques for patients with bifurcated coronary artery lesions. Catheter Cardiovasc Interv. 2020. https://doi.org/10.1002/ ccd.29071.

4. Song YB, Hahn JY, Yang JH, Choi SH, Choi JH, Lee SH, et al. Differential prognostic impact of treatment strategy among patients with left main versus non-left main bifurcation lesions undergoing percutaneous coronary intervention: results from the COBIS (Coronary Bifurcation Stenting) Registry II. JACC Cardiovase Interv. 2014;7:255-63.

5. Serruys PW, Morice MC, Kappetein AP, Colombo A, Holmes DR, Mack MJ, et al. Percutaneous coronary intervention versus coronary-artery bypass grafting for severe coronary artery disease. N Engl J Med. 2009;360:961-72.

6. Tiroch K, Mehilli J, Byrne RA, Schulz S, Massberg S, Laugwitz $\mathrm{K}$, et al. Impact of coronary anatomy and stenting technique on long-term outcome after drug-eluting stent implantation for unprotected left main coronary artery disease. JACC Cardiovasc Interv. 2014;7:29-36.

7. Stone GW, Sabik JF, Serruys PW, Simonton CA, Généreux P, Puskas J, et al. Everolimus-eluting stents or bypass surgery for left main coronary artery disease. N Engl J Med. 2016;375:2223-35.

8. Chen SL, Sheiban I, Xu B, Jepson N, Paiboon C, Zhang JJ, et al. Impact of the complexity of bifurcation lesions treated with drugeluting stents: the DEFINITION study (Definitions and impact of complEx biFurcation lesIons on clinical outcomes after percutaNeous coronary IntervenTIOn using drug-eluting steNts). JACC Cardiovasc Interv. 2014;7:1266-76.

9. Steigen TK, Maeng M, Wiseth R, Erglis A, Kumsars I, Narbute I, et al. Randomized study on simple versus complex stenting of coronary artery bifurcation lesions: the nordic bifurcation study. Circulation. 2006;114:1955-61.
10. Hildick-Smith D, de Belder AJ, Cooter N, Curzen NP, Clayton TC, Oldroyd KG, et al. Randomized trial of simple versus complex drug-eluting stenting for bifurcation lesions: the British bifurcation coronary study: old, new, and evolving strategies. Circulation. 2010;121:1235-43.

11. Hildick-Smith D, Behan MW, Lassen JF, Chieffo A, Lefèvre T, Stankovic G, et al. The EBC TWO study (European Bifurcation Coronary TWO): a randomized comparison of provisional T-stenting versus a systematic 2 stent culotte strategy in large caliber true bifurcations. Circ Cardiovasc Interv. 2016;9(9):e003643.

12. Kumsars I, Holm NR, Niemelä M, Erglis A, Kervinen K, Christiansen EH, et al. Randomised comparison of provisional side branch stenting versus a two-stent strategy for treatment of true coronary bifurcation lesions involving a large side branch: the Nordic-Baltic Bifurcation Study IV. Open Heart. 2020;7(1):e000947.

13. Chen SL, Zhang JJ, Han Y, Kan J, Chen L, Qiu C, et al. Double kissing crush versus provisional stenting for left main distal bifurcation lesions: DKCRUSH-V randomized trial. J Am Coll Cardiol. 2017;70:2605-17.

14. Toyofuku M, Kimura T, Morimoto T, Hayashi Y, Shiode N, Nishikawa $\mathrm{H}$, et al. Comparison of 5-year outcomes in patients with and without unprotected left main coronary artery disease after treatment with sirolimus-eluting stents: insights from the j-Cypher registry. JACC Cardiovasc Interv. 2013;6:654-63.

15. Ohya M, Kadota K, Toyofuku M, Morimoto T, Higami H, Fuku Y, et al. Long-term outcomes after stent implantation for left main coronary artery (from the Multicenter Assessing Optimal Percutaneous Coronary Intervention for Left Main Coronary Artery Stenting Registry). Am J Cardiol. 2017;119:355-64.

16. Lee PH, Kwon O, Ahn JM, Lee CH, Kang DY, Lee JB, et al. Safety and effectiveness of second-generation drug-eluting stents in patients with left main coronary artery disease. J Am Coll Cardiol. 2018;71:832-41.

17. Song PS, Song YB, Lee JM, Hahn JY, Choi SH, Choi JH, et al. Major predictors of long-term clinical outcomes after percutaneous coronary intervention for coronary bifurcation lesions with 2-stent strategy: patient-level analysis of the Korean bifurcation pooled cohorts. JACC Cardiovasc Interv. 2016;9:1879-86.

18. Cho S, Kang TS, Kim JS, Hong SJ, Shin DH, Ahn CM, et al. Long-term clinical outcomes and optimal stent strategy in left main coronary bifurcation stenting. JACC Cardiovasc Interv. 2018;11:1247-58.

19. Ohya M, Morimoto T, Kubo S, Habara S, Kuwayama A, Miura $\mathrm{K}$, et al. Two-year outcomes and predictors of target lesion revascularization for non-left main coronary bifurcation lesions following two-stent strategy with 2nd-generation drug-eluting stents. Circ J. 2018;82:798-806.

20. Sawaya FJ, Lefèvre T, Chevalier B, Garot P, Hovasse T, Morice $\mathrm{MC}$, et al. Contemporary approach to coronary bifurcation lesion treatment. JACC Cardiovasc Interv. 2016;9:1861-78.

21. Hikichi Y, Inoue T, Node K. Benefits and limitations of cypher stent-based bifurcation approaches: in vitro evaluation using micro-focus CT scan. J Interv Cardiol. 2009;22:128-34.

22. Murasato Y. Bench Testing of Coronary Bifurcation Stenting Techniques: How Is It Done? Does It Help Technical Decision Making? Tips and Tricks in Interventional Therapy of Coronary Bifurcation Lesions. Edited by Moussa I and Colombo A. 2010;192-210. Informa Healthcare, London

23. Chen SL, Xu B, Han YL, Sheiban I, Zhang JJ, Ye F, et al. Clinical outcome after DK crush versus culotte stenting of distal left main bifurcation lesions: the 3-year follow-up results of the DKCRUSH-III study. JACC Cardiovasc Interv. 2015;8:1335-42.

24. Chen E, Cai W, Chen LL. Crush versus Culotte stenting techniques for coronary bifurcation lesions: a systematic review 
and meta-analysis of clinical trials with long-term follow-up. Medicine (Baltimore). 2019;98(14):e14865.

25. Dérimay F, Finet G, Souteyrand G, Maillard L, Aminian A, Lattuca $\mathrm{B}$, et al. Benefit of a new provisional stenting strategy, the re-proximal optimisation technique: the rePOT clinical study. EuroIntervention. 2018;14:e325-32.

26. Murasato Y, Mori T, Okamura T, Nagoshi R, Fujimura T, Yamawaki M, et al. Efficacy of the proximal optimization technique on crossover stenting in coronary bifurcation lesions in the 3D-OCT bifurcation registry. Int J Cardiovasc Imaging. 2019;35:981-90.

27. Kume T, Yamada R, Koyama T, Tamada T, Imai K, Fukuhara $\mathrm{K}$, et al. Coronary bifurcation bench test using multimodality imaging: Impact of stent strut link location on stent deformity and jailed side-branch orifices during re-proximal optimizing technique. Catheter Cardiovasc Interv. 2019;93:E17-23.

28. Takagi K, Nagoshi R, Kim BK, Kim W, Kinoshita Y, Shite J, et al. Efficacy of coronary imaging on bifurcation intervention. Cardiovasc Interv Ther. 2020. https://doi.org/10.1007/s12928-020-00701 -2 .

29. Murasato Y, Nishihara M, Mori T, Meno K, Shibao K, Takenaka $\mathrm{K}$ et al. Feasibility and efficacy of the ultrashort side branch dedicated balloon in coronary bifurcation stenting. EuroIntervention. 2020:EIJ-D-20-00334.

30. Koo BK, Kang HJ, Youn TJ, Chae IH, Choi DJ, Kim HS, et al. Physiologic assessment of jailed side branch lesions using fractional flow reserve. J Am Coll Cardiol. 2005;46:633-7.

31. Koo BK, Park KW, Kang HJ, Cho YS, Chung WY, Youn TJ, et al. Physiological evaluation of the provisional side-branch intervention strategy for bifurcation lesions using fractional flow reserve. Eur Heart J. 2008;29:726-32.

32. Lee CH, Choi SW, Hwang J, Kim IC, Cho YK, Park HS, et al. 5 -year outcomes according to FFR of left circumflex coronary artery after left main crossover stenting. JACC Cardiovasc Interv. 2019; 12:847-55.

33. Foin N, Torii R, Mortier P, De Beule M, Viceconte N, Chan PH, et al. Kissing balloon or sequential dilation of the side branch and main vessel for provisional stenting of bifurcations: lessons from micro-computed tomography and computational simulations. JACC Cardiovasc Interv. 2012;5:47-56.

34. Takahashi H, Otake H, Shinke T, Murasato Y, Kinoshita Y, Yamawaki M, et al. Impact of final kissing balloon inflation on vessel healing following drug-eluting stent implantation: insight from the optical coherence tomography sub-study of the J-REVERSE trial. J Cardiol. 2016;68:504-11.

35. Fujino Y, Attizzani GF, Tahara S, Naganuma T, Takagi K, Yabushita $\mathrm{H}$, et al. Difference in vascular response between sirolimus-eluting- and everolimus-eluting stents in ostial left circumflex artery after unprotected left main as observed by optical coherence tomography. Int J Cardiol. 2017;230:284-92.

36. Okamura T, Nagoshi R, Fujimura T, Murasato Y, Yamawaki $\mathrm{M}$, Ono $\mathrm{S}$, et al. Impact of guidewire recrossing point into stent jailed side branch for optimal kissing balloon dilatation: core lab 3D optical coherence tomography analysis. EuroIntervention. 2018;13:e1785-93.

37. Fujino Y, Attizzani GF, Tahara S, Takagi K, Naganuma T, Wang $\mathrm{W}$, et al. Impact of main-branch calcified plaque on side-branch stenosis in bifurcation stenting: an optical coherence tomography study. Int J Cardiol. 2014;176:1056-60.

38. Maejima N, Hibi K, Saka K, Akiyama E, Konishi M, Endo $\mathrm{M}$, et al. Relationship between thickness of calcium on optical coherence tomography and crack formation after balloon dilatation in calcified plaque requiring rotational atherectomy. Circ J. 2016;80:1413-9.

39. Fujino A, Mintz GS, Matsumura M, Lee T, Kim SY, Hoshino M, et al. A new optical coherence tomography-based calcium scoring system to predict stent underexpansion. EuroIntervention. 2018;13:e2182-9.

Publisher's Note Springer Nature remains neutral with regard to jurisdictional claims in published maps and institutional affiliations. 